\title{
The Humphrey optical coherence tomography scanner: quantitative analysis and reproducibility study of the normal human retinal nerve fibre layer
}

\author{
Adrian L Jones, Nick J L Sheen, Rachel V North, James E Morgan
}

\begin{abstract}
Backgroundlaims-To determine the reproducibility of the Humphrey optical coherence tomography scanner (OCT), software version 5.0 , for measurement of retinal nerve fibre layer (RNFL) thickness in normal subjects and to compare OCT measurements with published histological thickness of the human RNFL.

Methods-Three independent measurements were obtained at each session for one eye from 15 normal subjects with a mean age of 30.8 (SD 10.9) years. Scans were taken in the peripapillary retina using the default setting $(1.74 \mathrm{~mm}$ radius from centre of the optic disc) and were repeated 1 week later. Additional scans were obtained at the optic nerve head (ONH) margin overlying the scleral rim, for comparison with available histological data on the human RNFL.
\end{abstract}

Results-For the $1.74 \mathrm{~mm}$ circular scan, the mean coefficient of variation (COV) for the global RNFL thickness measurement was $5 \%$ (SD 3\%). This increased to $8 \%(3 \%)$ for quadrant measurements and to $9 \%(3 \%)$ with further subdivision into 12 segments. Significant differences $(p<0.05)$ between sessions were only found when the data were divided into segments. The mean RNFL thickness for the 1.74 mm scan was $127.87(9.81) \mu \mathrm{m}$. The RNFL was maximal at the superior disc pole, $161.44 \mu \mathrm{m}$ (14.8), and minimal at the temporal pole, $83.1(12.8) \mu \mathrm{m}$. Peak thickness values occurred superior temporal and inferior temporal to the vertical axis. RNFL thickness for every sector of the disc was greatest at the margin of the optic disc (mean $185.79 \mu \mathrm{m}$; SD 32.61). Although the variation in RNFL thickness around the disc follows published histology data, the OCT underestimates RNFL thickness by an average of $37 \%$ (SD 11; range $21-48 \%$ ).

Conclusion-The OCT provides reproducible measurement of the retinal structures that are consistent with the properties of the RNFL. However, comparison with available studies of RNFL thickness in the human suggests that in its present form, the OCT underestimates RNFL thickness. Further refinement of this technology is required to improve the accuracy with which the OCT measures retinal nerve fibre layer thickness. (Br f Ophthalmol 2001;85:673-677)
Optical coherence tomography (OCT) is a new technique for the in vivo acquisition of cross sectional images of retinal structure, from which clinically relevant thickness estimates of the retinal layers can be made. The theoretical axial resolution of OCT is approximately 14 $\mu \mathrm{m}$ in air and $10 \mu \mathrm{m}$ in optically clear biological tissues, ${ }^{1}$ which is a significant improvement over the resolution of approximately $300 \mu \mathrm{m}$ for the scanning laser ophthalmoscope. ${ }^{2}$

The principles of image acquisition have been described in detail elsewhere. ${ }^{134}$ In brief, the OCT uses low coherence interferometry to provide absolute measurements of retinal thickness, thereby eliminating the need for a reference plane. ${ }^{5}$ Comparison of histological and OCT images from prototype devices have shown a good correlation between the OCT estimates and real measurements of retinal nerve fibre layer (RNFL) thickness. ${ }^{6-9}$ OCT has been developed for use in the clinical setting and shows particular promise in the assessment of macular pathology, with OCT images showing a close correlation with the histological structure of macular holes, ${ }^{1011}$ cystoid macular oedema, ${ }^{12}$ central serous retinopathy, ${ }^{13}$ retinal detachment, ${ }^{14}$ choroidal neovascularisation, ${ }^{15}$ and retinal trauma. ${ }^{16} \mathrm{Com}-$ pared with prototype versions, parameters such as laser wavelength and intensity have been changed in the commercial version of the OCT that would be expected to alter the lasertissue interaction and provide different estimates of retinal structure. While this may not be a critical issue when providing qualitative assessment of macular pathology it is important in the quantitative assessment of retinal structures such as nerve fibre layer thickness in the assessment of glaucoma. ${ }^{4517-21}$ In this study we have therefore evaluated the reproducibility of the Humphrey OCT and have compared estimates of RNFL thickness obtained using the latest software version with published histological measurements of the human RNFL.

Materials and methods

SUBJECT CHARACTERISTICS

Subjects were recruited from staff and students of the Department of Optometry and Vision Sciences. Written informed consent was obtained in compliance with ethical standards of the Bro Taf Community Health Authority. Each subject undertook a complete ophthalmic examination, which included anterior segment and fundus biomicroscopy, refraction, keratometry, automated static perimetry, and 

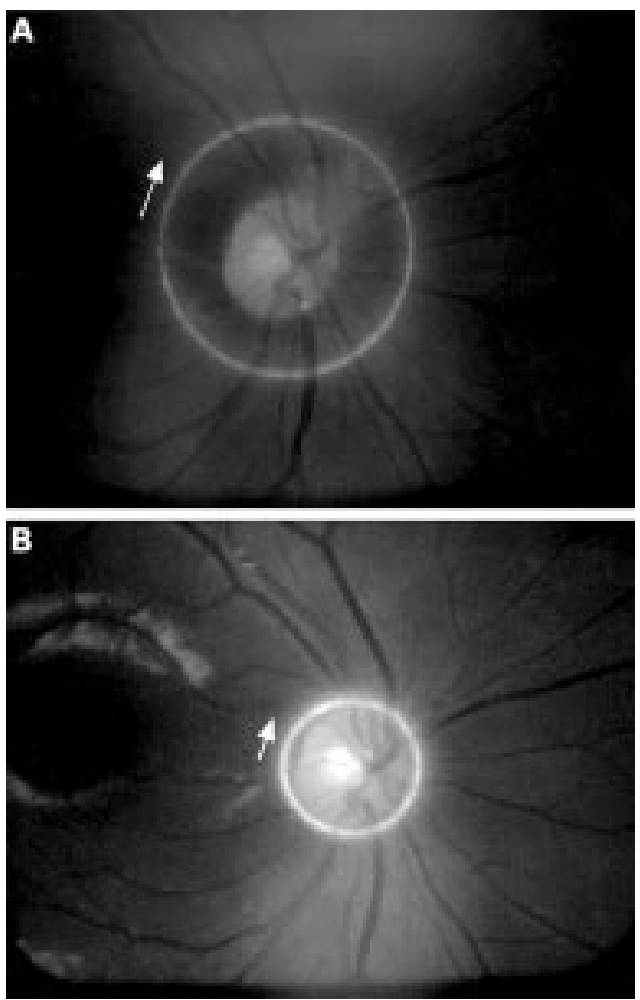

Figure 1 Operator monitor images showing the scan patterns with indication of scan direction (RE), (A) 1.74 mm circle scan, (B) ONH margin scan.

tonometry. Subjects were classified as having normal eyes if they had no history or evidence of ocular pathology or surgery (including refractive), absence of visual field defects (Humphrey 24-2 SITA-Standard), best corrected visual acuity of $6 / 9$ or greater, and intraocular pressures of $21 \mathrm{~mm} \mathrm{Hg}$ or less (Goldmann). The pupil of each subject's nondominant eye was dilated using $1 \%$ tropicamide topical drops to give a minimum pupil diameter of $7 \mathrm{~mm}$.

OCT IMAGE ACQUISITION

All images were acquired by a single experienced observer using the Humphrey OCT Model 2000, Humphrey Instruments, CA, USA (software V 5.0). The light source in the OCT is a superluminescent diode with a wavelength of $850 \mathrm{~nm}$, the power was $750 \mu \mathrm{W}$ and the image acquisition time was approximately 1 second for each scan. OCT analysis was performed along a line consisting of 100 axial

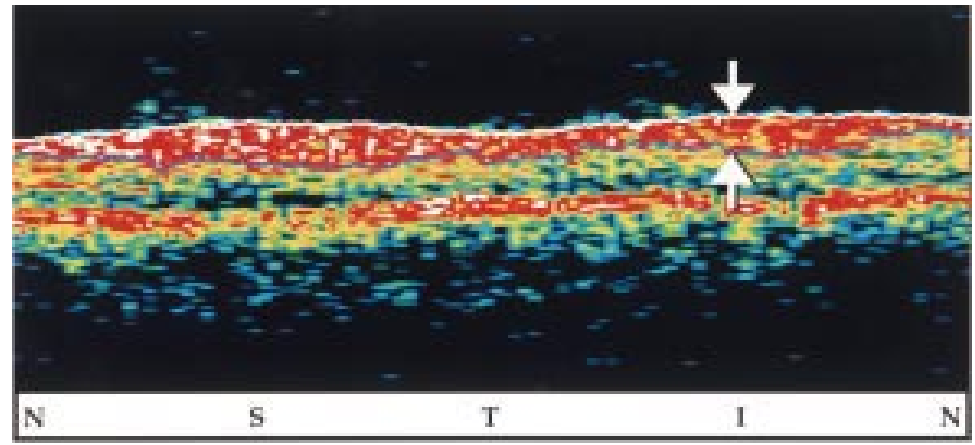

Figure 2 Raw data image as supplied by the commercial OCT software, the arrows indicate the RNFL. $N=$ nasal, $S=$ superior, $T=$ temporal, $I=$ inferior. scans. Since the number of axial scans is fixed, the sample density varied as an inverse function of scan length. An internal fixation target offset nasally from the scan area has previously been shown to give the highest reproducibility ${ }^{22}$ and was therefore used for all image acquisition. For each subject the image of the $\mathrm{ONH}$ was focused and aligned using the real time video monitor. Three ${ }^{23}$ circular scans were taken around the optic nerve head at the default radius of $1.74 \mathrm{~mm}$ from the centre of the optic disc. ${ }^{22}$ The coordinates of the retinal landmarks were recorded together with the scan and fixation coordinates. The procedure was repeated 1 week later.

In a separate session, a single set of three scans was obtained from the margin of the optic disc as defined by the location of Elshnig's ring. Images were rejected if significant eye movement occurred during the scan.

The OCT software generates a colour coded image for the display of the interferometric signals. Estimates of RNFL thickness are made from these signals using proprietary algorithms within the OCT software.

For each scan, the global RNFL thickness was determined from 100 points around the disc. The mean RNFL thickness was also calculated for four 90 degree quadrants and twelve 30 degree segments by the OCT software. Statistical analysis was performed using sPSS V.8. (SPSS Inc, Chicago, IL, USA). Intersession variation was assessed using the Friedman test and intrasession variation using the Wilcoxon test. Coefficients of variation for segment, quadrant, and global values were calculated for each subject.

\section{Results}

OCT examinations were performed on 15 eyes of 15 subjects (five male, 10 female) with a mean age of 30.80 years (SD 10.86; range 20-53 years). The mean spherical refractive error was -1.18 DS $(1.88 ;+0.50$ to $-5.50 \mathrm{DS})$ and mean astigmatic error was $0.45 \mathrm{DC}(0.37$; 0 to $1.00 \mathrm{DC})$. Good quality images were acquired in every case. Videographic images of the region of interest are shown in Figure 1, indicating the scan pattern at the default setting ( $1.74 \mathrm{~mm}$ radius) and at the disc margin; the cross sectional image of peripapillary retina is shown in Figure 2.

The mean RNFL thickness for the $1.74 \mathrm{~mm}$ radius was $127.87 \mu \mathrm{m}$ (SD 9.81). The mean standard deviation was $6.33(3.52) \mu \mathrm{m}$ with a mean COV of $5 \%$ (3\%). When RNFL thickness was considered in quadrants (superior, inferior, nasal and temporal) these values increased to $9.69(2.05) \mu \mathrm{m}$ and $8 \%(3 \%)$ respectively, increasing again to 11.33 (1.80) $\mu \mathrm{m}$ and $9 \%(3 \%)$ when RNFL thickness was further subdivided into 12 segments. Figure 3 shows the RNFL thickness plotted for both sessions and the COV calculated from each subject for every segment (range 5-20\%). The nasal areas of the RNFL provided the least reliable measurements with mean COV value of $20 \%$; however, this did not reach significance $(p>0.05)$. When the RNFL thickness 
measurements at this eccentricity were combined for the first and second imaging sessions the mean was found to be $127.79(0.31) \mu \mathrm{m}$. Intersession significant differences were only found for the segment values $(\mathrm{p}<0.05)$. Those subjects with significant intersession differences tended to have lower mean RNFL thickness values.

For OCT scans taken at the margin of the optic disc, the mean RNFL thickness was 185.79 (32.61) $\mu \mathrm{m}$ with a mean standard

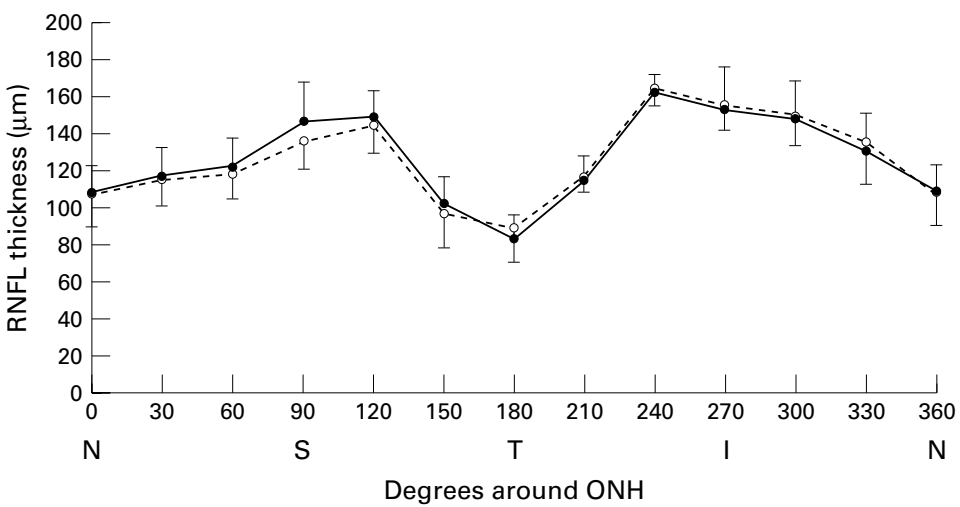

Figure 3 Plot of the mean RNFL thickness per segment for each session using the 1.74 $m m$ circle scan, error bars indicate $1 S D$ from the mean. Open circles represent values from session 1; solid circles from session $2 . N=$ nasal, $S=$ superior, $T=$ temporal, $I=$ inferior.

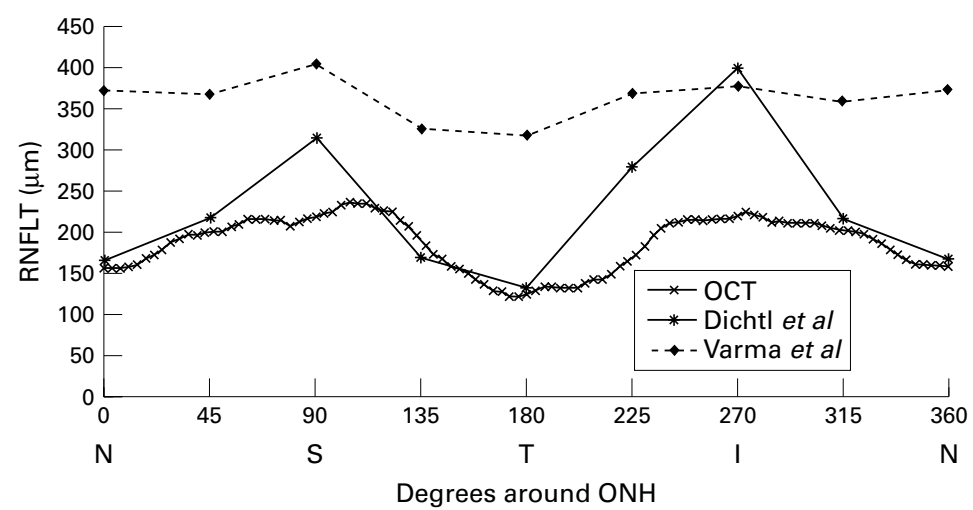

Figure 4 Plot of RNFL thickness according to angular location around the optic disc circumference. $N=$ nasal, $S=$ superior, $T=$ temporal, $I=$ inferior. Plots marked "OCT" represent values obtained by the OCT scan at the ONH margin. Those marked "Dichtl et al" and "Varma et al" represent RNFL thickness at the ONH margin as published by Dichtl et al,,$^{36}$ and Varma et al, ${ }^{35}$ respectively. Note the difference in modulation of the RNFL as measured by histology.

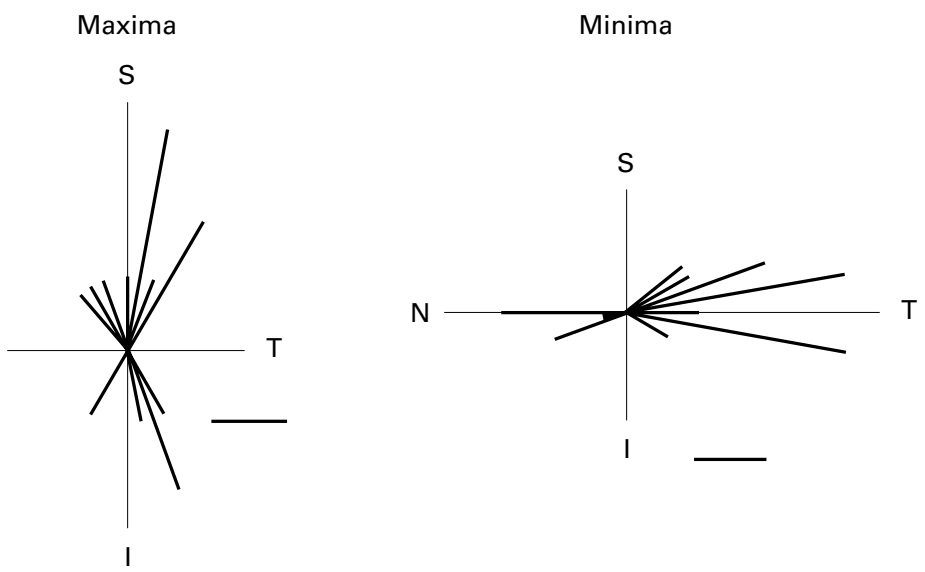

Figure 5 Polar frequency histogram showing the distribution of the maxima and minima for RNFL thickness at the ONH margin as measured by the OCT. $N=$ nasal, $S=$ superior, $T=$ temporal, $I=$ inferior. Scale bar shows representation for one subject. deviation of $44.61(7.48) \mu \mathrm{m}$. The variation in mean RNFL thickness as estimated by the OCT is shown in Figure 4. Published histological measurements of RNFL thickness from two studies have been overlaid for comparison, which are greater at every point around the ONH. In Figure 5 the location of the peak RNFL thickness has been represented as a polar histogram, showing that peak RNFL thickness occurred at the superior and inferior poles with a skew to the temporal aspect of the disc.

\section{Discussion}

Our results are generally in agreement with previous studies using prototype OCT devices that report mean peripapillary RNFL thickness in the range of $80-150 \mu \mathrm{m},{ }^{41722}{ }^{24-30}$ with mean standard deviations of $3-20 \mu \mathrm{m}^{27}{ }^{28}$ when using the $1.74 \mathrm{~mm}$ radius scan. Our mean COVs are also similar to those obtained earlier studies of $7.5 \%$ to $20.2 \%,{ }^{30} 31$ although the mean standard deviations are larger than those recorded previously of $(6.33 v 2.8 \mu \mathrm{m}) .{ }^{27}{ }^{28} \mathrm{As}$ with these studies, we have shown that COV increases with increasing subdivision of peripapillary RNFL thickness measurements and that it varies around the optic disc, being highest in the nasal retina and lowest at the superior and inferior poles. Studies with other imaging devices such as scanning laser polarimeters ${ }^{32} 33$ and confocal scanning laser tomographer ${ }^{34}$ give similar COV values, suggesting that a change of at least $20 \mu \mathrm{m}$ in the global RNFL thickness would be required to produce a clinically significant change in the OCT measurements.

An important finding of the present study is that even with the latest RNFL algorithm (software V 5.0), the Humphrey OCT underestimates the RNFL thickness when compared with histological data. ${ }^{35}{ }^{36}$ It can be seen from Figure 4 that the OCT on average gives only $63 \%$ (range $52 \%-79 \%$ ) of the histological RNFL thickness. Assuming 10-15\% shrinkage for histological preparation, ${ }^{35}$ it is possible the OCT could be underestimating RNFL thickness by as much as $47 \%$. The discrepancy may increase further when account is taken of the age between the subjects in this study. Significant age related thinning of the human RNFL has been reported in both histological ${ }^{35}{ }^{36}$ studies and with other imaging devices. ${ }^{24}$

Entire retinal thickness measurements (rather than RNFL thickness) have been shown to provide greater reproducibility ${ }^{37}$ and better agreement with human histology. ${ }^{638}$ This may be due to a greater change in refractive index, and hence reflectance, at the retinal pigment epithelial/photoreceptor interface than between that of the retinal ganglion cell/retinal nerve fibre layer, thereby providing a higher definition of tissue boundary. This study has not addressed the method by which the OCT software calculates RNFL thickness from the digital interference signal recorded by the Michaelson interferometer within the OCT hardware. These values are displayed on the monitor using a proprietary colour palette to represent the digital values. Correlation of the 
OCT interference signals with retinal histology has to date been mostly empirical and is currently under investigation in our laboratory.

Comparison of the variation in RNFL thickness around the $\mathrm{ONH}$ is also instructive. One of the problems in drawing comparisons with histological measurements of RNFL thickness in the human is that existing reports do not provide a consistent picture (Fig 4). It can be seen that the OCT measurements show better agreement with data from Varma et $a l^{35}$ in that it displays a more gradual modulation in RNFL thickness. Within the literature, the profile of the peripapillary RNFL thickness has been suggested to follow a "double hump" configuration. However, the degree to which this is shown by the various imaging devices is inconsistent. For example, a recent study with confocal scanning laser ophthalmoscopy (Heidelberg retina tomograph; Heidelberg Engineering $\mathrm{GmbH}$, Heidelberg, Germany) ${ }^{39}$ suggested a much lower modulation of the RNFL profile that was more consistent with Varma et $a l .{ }^{35}$ Other studies using computer topographic analysis of simultaneous stereoscopic videographic images (Rodenstock Analyser; Rodenstock Instruments, Munich, Germany) ${ }^{39}$ and scanning laser polarimetry (Nerve Fibre Layer Analyser; Laser Diagnostic Technologies, San Diego, CA, USA ${ }^{40}$ reported modulation similar to that found by Dicht et al. ${ }^{36}$ In the present study, the peak RNFL thickness was most frequently located along the vertical meridian with slight skew to the temporal aspect of the ONH. Minimum RNFL thickness was also located most frequently at temporal pole of the $\mathrm{ONH}$, providing a greater relative change in RNFL thickness between the vertical and the temporal meridians of the $\mathrm{ONH}$ than between the vertical and nasal meridians.

A further artefact to consider is the shape of the scan used in comparison with the shape of the human ONH. In this study we utilised a circle scan, but since the $\mathrm{ONH}$ is elliptical in shape the information gathered from the temporal and nasal areas would be further from the $\mathrm{ONH}$ margin than the information from the poles. This would give lower RNFL thickness values for the temporal and nasal quadrants, thus artificially increasing the overall modulation in RNFL thickness. This suggests less modulation in the RNFL thickness at the nasal pole, and implies that the RNFL thickness profile of the human retina is more similar to that provided by Varma et al. ${ }^{35}$ Further work is needed to verify the modulation of RNFL thickness in the human retina.

Although the OCT data are consistent with the reduction in RNFL thickness with increasing eccentricity, this does not appear to be symmetrical when comparing the superior and inferior poles of the ONH. In several studies ${ }^{41}$ this can be expressed as the ratio of RNFL thickness superior to the $\mathrm{ONH}$ to that of the $\mathrm{RNFL}$ inferior to the $\mathrm{ONH}$ at the same eccentricity (S/I ratio). At the ONH margin, the S/I ratio was approximately 1.0 ; by contrast, in the peripapillary retina $(1.74 \mathrm{~mm})$ this had increased to 1.26. This implies a tendency for a greater relative RNFL thickness at the superior pole; however, this difference did not reach significance. This is in contrast with results obtained using other topographic devices at similar eccentricities from the $\mathrm{ONH}$ margin ${ }^{42}$ and the clinical evaluation of the neuroretinal $\operatorname{rim}^{43}$ where the inferior aspect often provides a thicker RNFL. It is possible that this results from subtle differences in axon trajectory away from the $\mathrm{ONH}$ and emphasises the importance of eccentricity when comparing RNFL thickness around the $\mathrm{ONH}$.

The OCT provides reproducible cross sectional images of the human retina from which estimates of RNFL and total retinal thickness can be made. These measurements may be of clinical value in the management of diseases such as glaucoma. Our study suggests that the current version of the OCT consistently underestimates RNFL thickness. In view of the considerable clinical potential of this technology, further work is required to improve the accuracy with which it measures retinal structure.

1 Huang D, Swanson EA, Lin CP, et al. Optical coherence tomography. Science 1991;254:1178-81.

2 Zinser G, Harbarth U, Schroder H. Formation and analysis of three dimensional data with laser tomographic scanner. In: Nasemann J, Burk R, eds. Scanning laser ophthalmoscopy and topography. Munich: Quintessenz, 1990.

3 Ripandelli G, Coppe AM, Capaldo A, et al. Optical coherence tomography. Sem Ophthalmol 1998;13:199-202.

4 Schuman JS, Hee MR Puliafito CA, et al. Quantification of chuma J , Hee MR, Puliafito CA, et al. Quantification of nerve fibre layer thickness in normal and glaucomatous eyes using optical

5 Pieroth L, Schuman JS, Hertzmark E, et al. Evaluation of focal defects of the nerve fibre layer using optical coherence tomography. Ophthalmology 1999;106:570-9.

6 Chauhan DS, Marshall J. The interpretation of optical coherence tomography images of the retina. Invest Ophthalmol Vis Sci 1999;40:2332-42.

7 Huang LN, Schuman JS, P-Kloizman T, et al. The comparison of nerve fiber layer thickness in glaucomatous monkey eyes measured by optical coherence tomography and histomorphometry. Invest Ophthalmol Vis Sci 1997;38: S838

8 Huang Y, Cideciyan AV, Papastergiou GI. Relation of optical coherence tomography to microanatomy in normal and rd chickens. Invest Ophthalmol Vis Sci 1998;39:2405-16.

9 Toth CA, Narayan DG, Boppart SA, et al. A comparison of retinal morphology viewed by optical coherence tomography and by light microscopy. Arch Ophthalmol 1997;115: graphy and

10 Hee MR, Puliafito CA, Wong C, et al. Optical coherence tomography of macular holes. Ophthalmology 1995;102: tomograp

11 Ripandelli G, Coppe AM, Bonini S, et al. Morphological evaluation of full-thickness idiopathic macular holes by optical coherence tomography. Eur f Ophthalmol 1999;9: $212-16$

12 Nussenblatt RB, Kaufman SC, Palestine AG, et al. Macular thickening and visual acuity. Measurement in patients with cystoid macular oedema. Ophthalmology 1987;94:1134-9.

13 Hee MR, Puliafito CA, Wong C, et al. Optical coherence tomography of central serous chorioretinopathy. $\mathrm{Am} \mathcal{F}$ Ophthalmol 1995;120:65-74.

14 Ip M, Garza-Karren C, Duker JS, et al. Differentiation of degenerative retinoschisis from retinal detachment using optical coherence tomography. Ophthalmology 1999;106: 600-5.

15 Giovannini A, Amato GP, Mariotti C, et al. OCT imaging of choroidal neovascularisation and its role in the determina-
tion of patients' eligibility for surgery. Br $\mathcal{F}$ Ophthalmol tion of patients'

16 Budde WM, Junemann A. Chalcosis oculi. Klin Monatsbl Augenheilkd 1998;212:184-5.

17 Bowd C, Weinreb RN, Williams JM, et al. The retinal nerve fiber layer thickness in ocular hypertensive, normal, and glaucomatous eyes with optical coherence tomography. Arch Ophthalmol 2000;118:22-6.

18 Burk R, Tuulomen A, Airaksinen PJ. Laser scanning tomography of localised nerve fibre layer defects. $\mathrm{Br} \mathcal{F}$ Ophthalmol 1998;82:1112-17.

19 Mistlberger A, Liebmann JM, Greenfield DS, et al. Heidelberg retina tomography and optical coherence tomography in normal, ocular-hypertensive, and glaucomatous eyes. in normal, ocular-hypertensive, and

20 Parisi V, Manni G, Gandolfi SA, et al. Visual function correlates with nerve fibre layer thickness in eyes affected by ocular hypertension. Invest Ophthalmol Vis Sci 1999;40: 1828-33. 
21 Schuman JS, Hee MR, Arya AV, et al. Optical coherence tomography: new tool for glaucoma diagnosis. Curr Opin tomography: a new tool fo

22 Schuman JS, Pedut-Kloizman T, Hertzmark E, et al. Reproducibility of nerve fibre layer thickness measurements using optical coherence tomography. Ophthalmology 1996;103: 1889-98.

23 Weinreb RN, Lusky M, Bartsch DW, et al. Effect of repetitive imaging on topographic measurements of the optic nerve head. Arch Ophthalmol 1993;111:636-8.

24 Chi QM, Tomita G, Inazumi K, et al. Evaluation of the effect of aging on the retinal nerve fibre layer thickness using scanning laser polarimetry. F Glaucoma 1995;4:406-13.

25 Gramer E, Dirmeyer M. Optical coherence tomography (OCT) to measure nerve fibre layer thickness in normal eyes. Invest Ophthalmol Vis Sci 1998;39:S933.

26 Hee MR, Izatt JA, Swanson EA, et al. Optical coherence tomography of the human retina. Arch Ophthalmol tomography of

27 Huang D, Hee MR, Pedut-Kloizman T, et al. A new algorithm for retinal nerve fibre layer thickness measurement by optical cohere

28 Nguyen R, Huang D, Hee MR, et al. Retinal nerve fiber layer distribution as measured by optical coherence tomography. Invest Ophthalmol Vis Sci 1996;37:S1096.

29 Walter A, Klemm M, Bernd AS, et al. Comparison and quantification of the retinal nerve fibre layer thickness with the nerve fiber analyser (NFA) and optical coherence tomography (OCT) in healthy subjects. Invest Ophthalmol Vis Sci 1997;38:S839.

30 Williams JM, Zangwill LM, Berry CC, et al. Reproducibility of nerve fibre layer thickness measurements using optical coherence tomography. Invest Ophthalmol Vis Sci 1999;40: S75.

31 Gurses-Ozden R, Ishikawa H, Hoh ST, et al. Increasing sampling density improves reproducibility of optical coherence tomography measurements. F Glaucoma 1999;8:238ence

32 Waid

Waldock A, Potts MJ, Sparrow JM, et al. Clinical evaluation of scanning laser polarimetry: 1 Intraoperator reproducibility and design of a blood vessel removal algorithm. $\mathrm{Br} F$ Ophthalmol 1998;82:252-9.

33 Hoh ST, Ishikawa H, Green field DS, et al. Peripapillary nerve fiber layer thickness measurement reproducibility using scanning laser polarimetry. F Glaucoma 1998;7:12-

34 Azuara-Blanco A, Harris A, Cantor LB. Reproducibility of optic disk topographic measurements with the Topcon ImageNet and the Heidelberg retina tomograph. Ophthalmologica 1998;212:95-8.

35 Varma R, Skaf M, Barron E. Retinal nerve fibre layer thickness in normal human eyes. Ophthalmology 1996;103: ness in nor.

36 Dichtl A, Jonas JB, Naumann GOH. Retinal nerve fiber layer thickness in human eyes. Graefes Arch Clin Exp Ophthalmol 1999;237:474-9

37 Koozekanani D, Roberts C, Katz SE, et al. Intersession repeatability of macular thickness measurements with the Humphrey 2000 OCT. Invest Ophthalmol Vis Sci 2000;41: 1486-91.

38 Baumann M, Gentile RC, Liebmann JM, et al. Reproducibility of retinal thickness measurements in normal eyes using optical coherence tomography. Ophthalmic Surg Lasers 1998;29:280-5.

39 Caprioli J, Park HJ, Ugurlu S, et al. Slope of the peripapillary nerve fibre layer surface in glaucoma. Invest Ophthalmol Vis Sci 1998;39:2321-8.

40 Morgan JE, Waldock A. Scanning laser polarimetry of the normal human retinal nerve fibre layer: a quantitative analysis. Am f Ophthalmol 2000;129:76-82

41 Tjon-Fo-Sang MJ, Lemij HG. Retinal nerve fiber layer measurements in normal black subjects as determined with scanning laser polarimetry. Ophthalmology 1998;105:7881 .

42 Morgan JE, Waldock A, Jeffery G, et al. Retinal nerve fibre layer polarimetry: histological and clinical comparison. $\mathrm{BrF}$ Ophthalmol 1998;82:684-90.

43 Jonas JB, Gusek GC, Naumann GOH. Optic disc, cup and neuroretinal rim size, configuration and correlations in normal eyes. Invest Ophthalmol Vis Sci 1988;29:1151-8. 